\title{
The Ultrastructural Basis for Synaptic Transmission between Primary Muscle Afferents and Neurons in Clarke's Column of the Cat ${ }^{1}$
}

\author{
B. WALMSLEY, ${ }^{2}$ E. WIENIAWA-NARKIEWICZ, ${ }^{3}$ AND M. J. NICOL
}

\author{
Experimental Neurology Unit, John Curtin School of Medical Research, Australian National University, Canberra, Australian Capital \\ Territory, Australia.
}

\begin{abstract}
The synaptic connection between primary muscle afferents and dorsal spinocerebellar tract (DSCT) neurons has been studied in an attempt to reveal some of the mechanisms underlying excitatory transmission in the mammalian central nervous system. Previous electrophysiological experiments have shown that the excitatory postsynaptic potentials (EPSPs) evoked DSCT neurons by impulses in a single muscle afferent fluctuate in amplitude. These fluctuations occur between discrete amplitudes which are separated by quantal increments. Two alternative hypotheses relate such a quantal increment to all-or-nothing transmitter release from either (1) an entire synaptic bouton or (2) an individual transmitter release site, given that a bouton may contain multiple release sites. The present study was undertaken primarily to gain ultrastructural evidence on these proposals. Electrodes filled with horseradish peroxidase (HRP) were used to label single identified group la afferent fibers and DSCT neurons in the lumbar spinal cord of anesthetized cats. HRP-labeled la synaptic boutons, and the contacts formed between HRP-labeled la boutons and the dendrites of a DSCT neuron labeled intracellularly with HRP, were examined in serial sections under the electron microscope. Group la boutons were found to contain multiple synaptic specializations, as evidenced by pre- and postsynaptic thickenings and presynaptic clusters of vesicles. Careful examination of a bouton in serial sections revealed each specialization as a separate structure. These observations support the proposal that synaptic transmission between group I muscle afferents and DSCT neurons occurs with discrete all-or-nothing EPSPs associated with transmitter release sites, rather than boutons per se.
\end{abstract}

Primary afferents from hindlimb muscle spindles and tendon organs make monosynaptic connections with dorsal spinocerebellar tract (DSCT) neurons, situated in Clarke's column (Mann, 1973). In a previous study (Tracey and Walmsley, 1982, 1984), the excitatory postsynaptic pulentials (EPSPs) evoked in DSCT neurons by impulses in a single primary muscle afferent were found to fluctuate in amplitude from trial to trial. These fluctuations occur between dis-

Received August 20, 1984: Revised February 19, 1985;

Accepted February 28, 1985

${ }^{1}$ We are grateful to Dr. R. E. W. Fyffe and Dr. E. G. Jones for comments and suggestions on this paper, and to Mrs. M. Wiechert for typing the manuscript.

${ }^{2}$ To whom correspondence should be addressed

${ }^{3}$ Present address: Department of Anatomy, Faculty of Medicine, The University of Calgary, 3300 Hospital Dr., N.W., Calgary, Albcrta, Canada T2N $4 N 1$. crete amplitudes separated by a quantal increment. In an earlier electrophysiological/anatomical study on single group la fiber EPSPs evoked in cat spinal motoneurons (Redman and Walmsley, 1981, $1983 \mathrm{a}, \mathrm{b}$ ), it was proposed that such a quantal increment is due to all-or-nothing transmitter release from a synaptic bouton. Light microscopic observations on the synaptic connections between horseradish peroxidase (HRP)-identified primary muscle afferents and DSCT neurons have revealed that these afferent boutons vary greatly in size, from $1 \times 1 \mu \mathrm{m}$ up to "giant" boutons of $20 \times 3 \mu \mathrm{m}$ (Tracey and Walmsley, 1984). Electron microscopic studies in Clarke's column have revealed the presence of several types of synaptic contacts, including giant boutons (Szentagothai and Albert, 1955; Rethelyi, 1970; Saito, 1974, 1979; Houchin et al., 1983). It was suggested that the giant boutons contain multiple transmitter release sites and that synaptic transmission between group I muscle afferents and DSCT neurons occurs with the quantal increments underlying EPSP amplitude fluctuations associated with these transmitter release sites (Tracey and Walmsley, 1984).

However, until the present study, no information has been available on the ultrastructure of identified terminals contacting DSCT neurons. We have employed electron microscopy to examine in detail the ultrastructure of group la synapses in Clarke's column and the synaptic contacts between la muscle afferents and DSCT neurons, both identified by intracellular labeling with HRP. A complete picture of the synaptic specializations contained within each bouton was constructed, using serial sections. The results indicate that some of the la synaptic terminals on DSCT neurons contain multiple transmitter release sites. These observations are taken as support for the proposed relationship between quantal EPSPs and individual transmitter release sites rather than synaptic boutons.

\section{Materials and Methods}

Fxperiments were performed on cats weighing 1.5 to $2.5 \mathrm{~kg}$. The cats were anesthetized with sodium pentobarbitone (35 $\mathrm{mg} / \mathrm{kg}$, i.p.) and main tained with supplementary doses (5 mg, i.v.). Mean arterial pressure and ond tidal $\mathrm{CO}_{2}$ wore monitorcd.

HRP labeling of axons and neurons. The following muscles were exposed in the left hindlimb: medial gastrocnemius, lateral gastrocnemius, soleus, and plantaris. The teridons of these muscles were separated and cut at their insertion to allow each muscle to be individually stretched. The cat was fixed in a rigid animal frame, and a laminectomy was performed from $L 7$ to $L 3$. the exposed hindlimb muscles and spinal cord were covered with pools of mineral oil, maintained at 35 to $37^{\circ} \mathrm{C}$ by infrared heating. Bipolar stimulating electrodes were placed on the sciatic nerve and its branches to the exposed muscles.

Glass microelectrodes containing $10 \% \mathrm{HRP}$ in $1 \mathrm{M} \mathrm{KCl}$ were inserted into the dorsal columns near the junction of $L .3$ and I 4 segments of the spinal cord, about $200 \mu \mathrm{m}$ lateral to the midline. On intracellular penetration of a primary muscle afferent, its conduction time was recorded, allowing a preliminary classification into group I or group II. Fibers were classified as group la if they had high dynamic sensitivity to passive stretch and a silent period during active muscle contraction. Once the afferent was identified, HRP was iontophoresed into the axon for up to an hour. Iorlophoresis of HRP was continued only while the intracellular action potential was greater 
than $10 \mathrm{mV}$. Good results were obtained when the charge transfer was in the range 100 to $400 \mathrm{nA} / \mathrm{min}$.

In the same experiments, intracellular recordings were made from DSCT neurons in Clarke's column at the level of $L 3$ and $L 4$ segments of the cord. Antidromic identification of DSCT neurons was performed by stimulation of the dissected dorsolateral fasciculus of the spinal cord at the C2 level (Houchin et al., 1983; Tracey and Walmsley, 1984). The dorsal columns were removed for approximately $2 \mathrm{~cm}$ to avoid stimulation spreading to them. Electrodes, filled with $10 \% \mathrm{HRP}$ in $1 \mathrm{M} \mathrm{KCl}$, were driven into Clarke's column in the spinal cord approximately 200 to $300 \mu \mathrm{m}$ from the midline. Only DSCT neurons receiving monosynaptic excitation from group I muscle afferents were injected with HRP (10 to $30 \mathrm{nA}$ for 10 to $40 \mathrm{~min}$ ).

Following a 2- to 5-hr post-injection survival tirne, the animal was perfused with $3 \%$ glutaraldehyde in phosphate buffer $(\mathrm{pH} 7.2)$. The spinal cord segments $L 3$ and $L 4$ were removed and post-fixed for about $10 \mathrm{hr}$. Parasagittal sections $(100 \mu \mathrm{m})$ of these segments were cut using a Vibratome and reacted for HRP using diaminobenzidene (DAB).

Light microscopy. Sections were serially mounted on gelatin-coated slides and cleared in alcohols and xylene to enable complete reconstruction of DSCT neurons (See Fig. 1) by light microscopy.

Electron microscopy. Sections immersed in glycerol were carefully examined under the light microscope. Structures filled with HRP reaction product were readily observed, even in the nondehydrated tissue. Small areas $(2 \times 1 \mathrm{~mm})$ containing stained fibers or neurons were cut from the sections and transferred to $0.1 \mathrm{M}$ phosphate buffer $(\mathrm{pH} \mathrm{7.4)}$, washed, and left for 2 to $3 \mathrm{hr}$ at $4^{\circ} \mathrm{C}$. Subsequently, the tissue was post-fixed in $2 \% \mathrm{O}_{\mathrm{s}} \mathrm{O}_{4}$, stained en bloc with $2 \%$ uranyl acetate, dehydrated in alcohol, and embedded in Spurr resin. Thin sections $(\sim 100 \mathrm{~nm})$ were cut with a diamond knife (Diatome) on a Reichert microtome, collected on parlodion-coated slot grids, and examined in a Phillips 301 electron microscope.

HRP-labeled structures were easily observed, and in the majority of cases staining was light enough not to obscure intracellular components. Criteria for identification of synapses were as follows: (1) thickening of the apposed cellular membranes; (2) accumulation of synaptic vesicles close to the presynaptic membrane; and (3) synaptic cleft. Scquential sections of cach labeled structure were examined and relevant sections were photographed.

The possibility that observed contacts were actually autapses rather than afferent contacts could be ruled out because the axons of DSCT neurons do not give rise to recurrent collaterals (Randic et al., 1981; Houchin et al., 1983; personal observation of HRP-labeled DSCT neurons). Such a possibility does exist, for example, with spinal motoneurons.

\section{Results}

Results were obtained from experiments on 22 cats weighing 1.5 to $2.5 \mathrm{~kg}$.

Light microscopy of DSCT neurons. In confirmation of earlier HRP studies (Houchin et al., 1983; Tracey and Walmsley, 1984), the dendritic lrees of DSCT neurons were very proluse and exlended over $3 \mathrm{~mm}$ in the rostrocaudal direction. Figure 1 shows a camera lucida reconstruction of a typical DSCT neuron receiving group I muscle afferent input. Many fine branchlets can be seen arising from both proximal and distal dendrites, in contrast to the DSCT neurons illustrated in the HRP study by Randic et al. (1981).

Electron microscopy of contacts between la fibers and DSCT neurons. A previous light microscopic study (Tracey and Walmsley, 1984) failed to observe contacts between a single HRP-labeled afferent fiber and intracellularly labeled DSCT neurons. We were, however, successful in obtaining some results in the present study.

Staining of both DSCT neurons and la fibers was sufficient to allow easy recognition without obscuring ultrastructural details. (In a number of experiments, a cobalt enhancement of the DAB-HRP reaction was used (Adams, 1977, 1981), but the resulting strong label usually obscured the fine details of terminals in the electron microscope.)

Since one of our primary interests was to investigate whether a single primary afferent bouton contained multiple synaptic specializations, it was essential to examine serial sections of each contact. (Otherwise, an irregularly shaped specialization would appear as multiple contacts if examined only in a single section.) Eight HRPlabeled la boutons were examined fully in serial sections under the electron microscope. Figures 2 to 6 illustrate connections between 

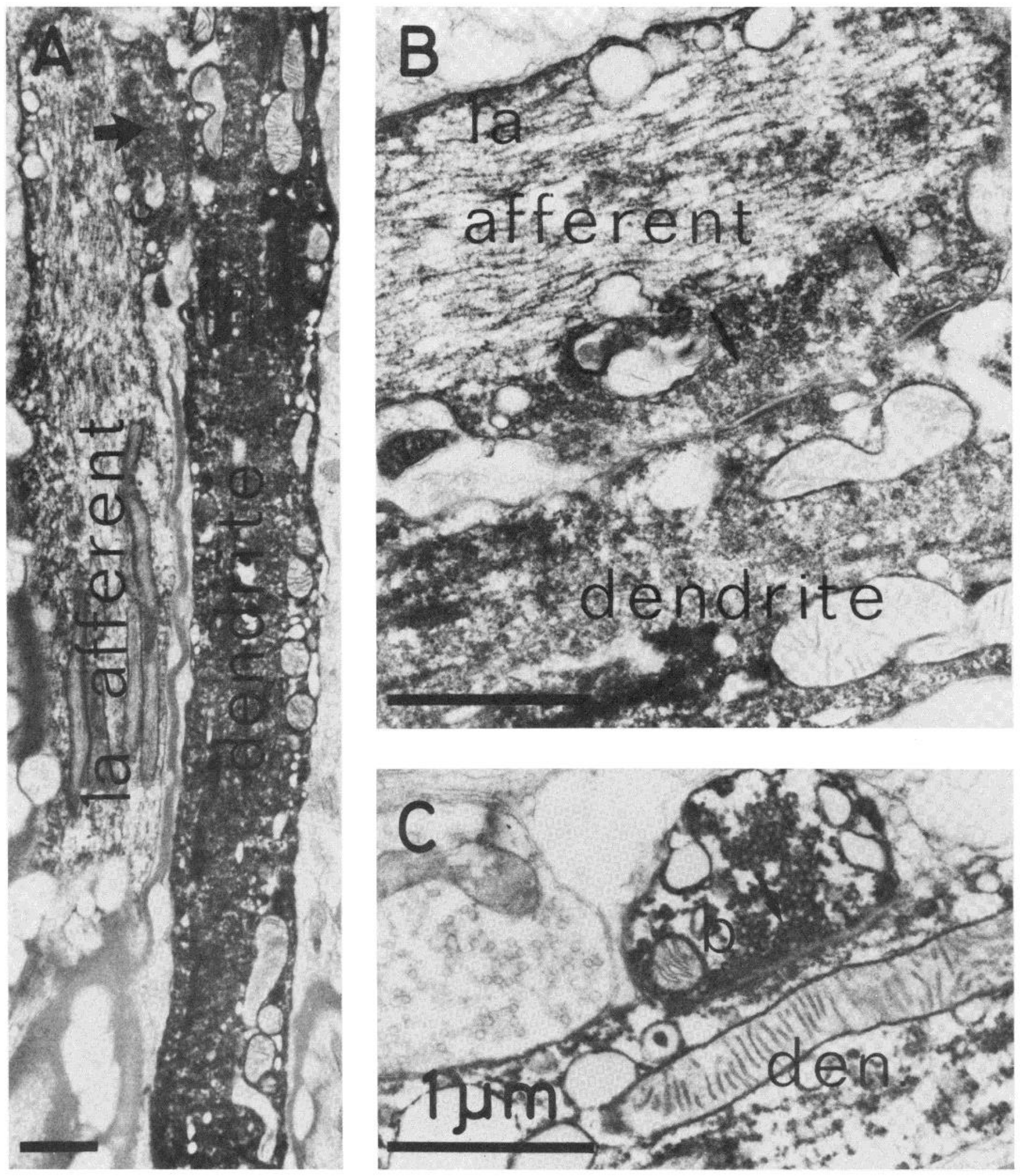

Figure 2. Electron micrographs of the connection between an HRP-stained la afferent and an HRP-stained DSCT neuron. An en passant connection between the la afferent and a dendrite of the DSCT neuron (arrow in $A$ ) is shown in more detail in $B$. The two arrows in $B$ indicate two synaptic specializations found at this connection. $C$ illustrates a contact (arrow) between a terminal la bouton and a dendrite (den.) of the DSCT neuron. 

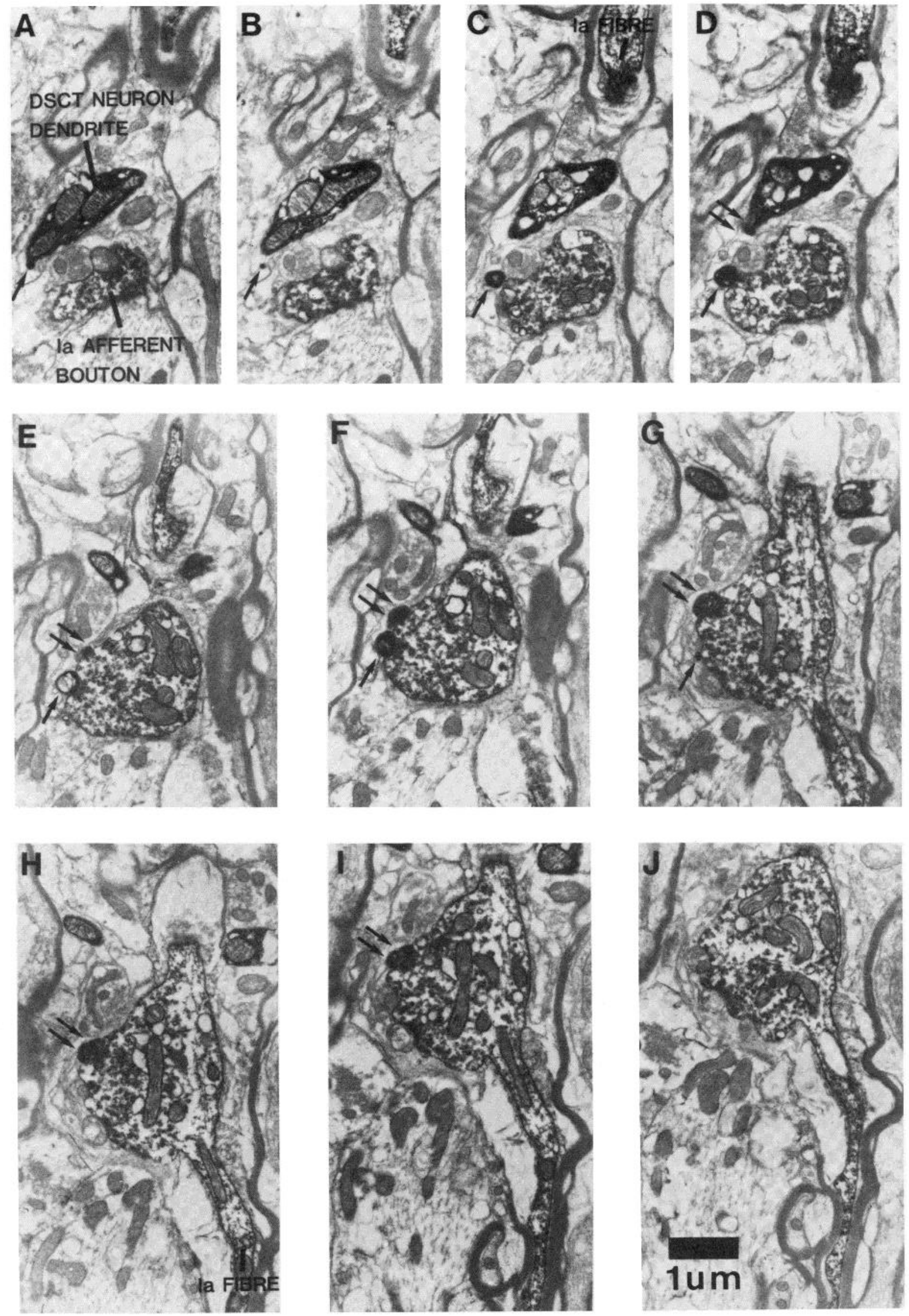

Figure 3. Serial sections through an HRP-labeled la bouton shown in sequential order, $A$ to $\mathrm{J}$. The la bouton made contact with two small branchlets (single and double arrows) which arise from a larger diameter dendrite of the HRP-labeled DSCT neuron. Details of the synaptic specializations in the la bouton are shown in Figure 4. 


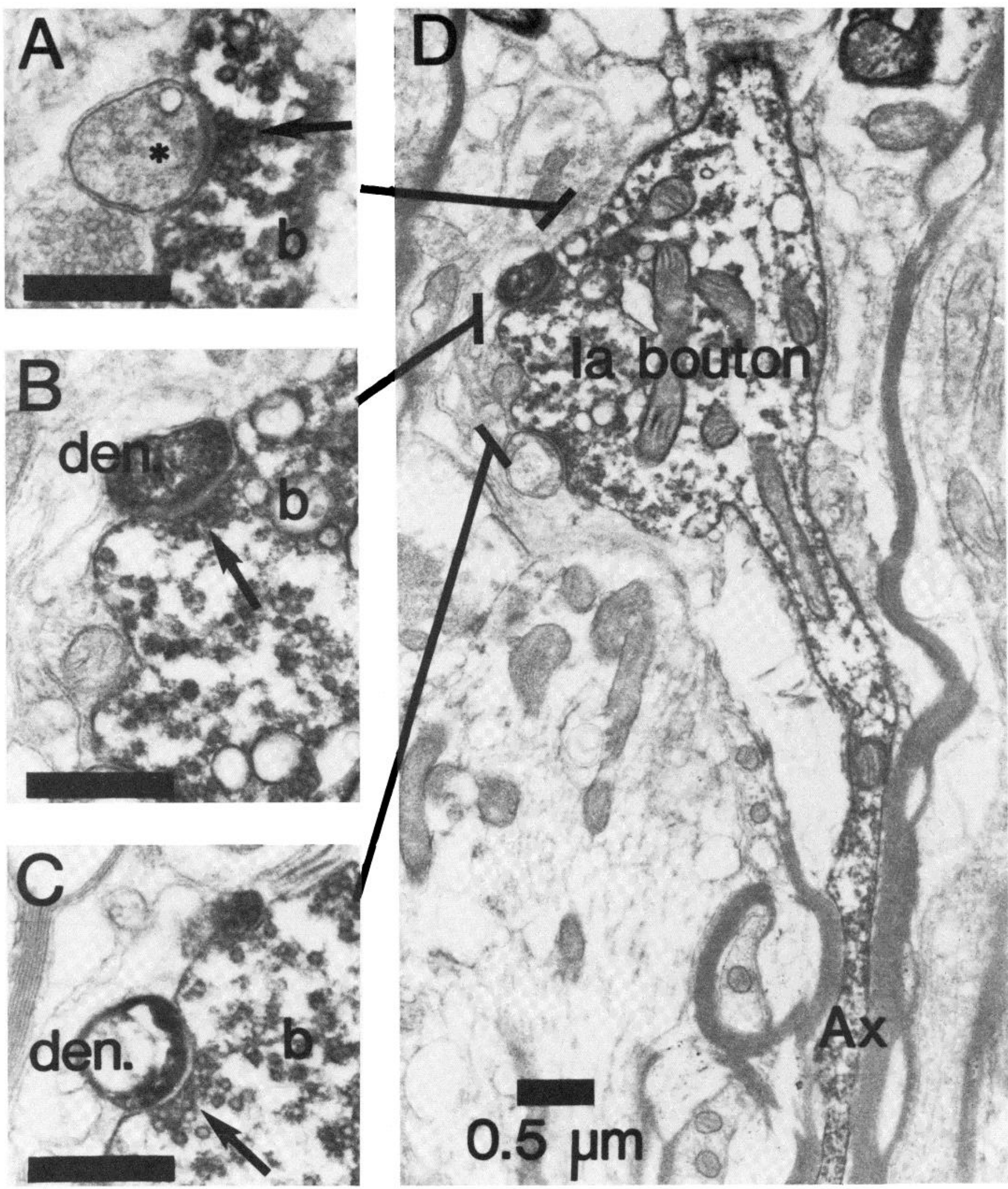

Figure 4. Contacts (arrows) formed by a la bouton $(D)$, labeled with HRP (same bouton as shown in Fig. 3). Two contacts were formed with different HRP-labeled dendrites (den. in $B$ and $C$ ), and a third contact was formed with an unlabeled structure (asterisk, in $A$ ). The approximate region in which these contacts were found is indicated by the lines joining the detailed photomicrographs $(A, B$, and $C)$ and the photomicrograph in $D$. Only the postsynaptic dendrite of $A$ can be seen in the single section in $D$. All calibration bars are $0.5 \mu \mathrm{m}$. 

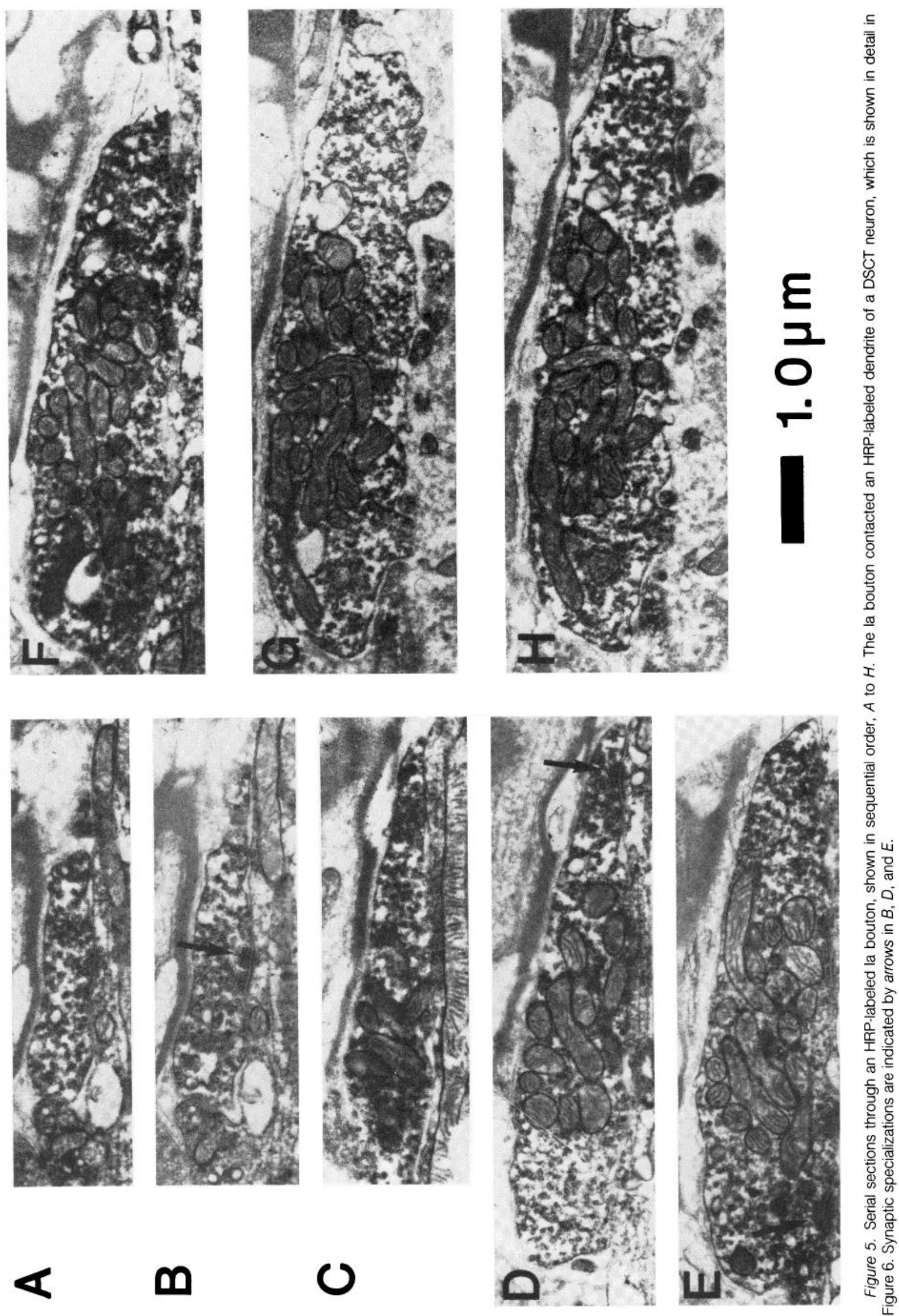


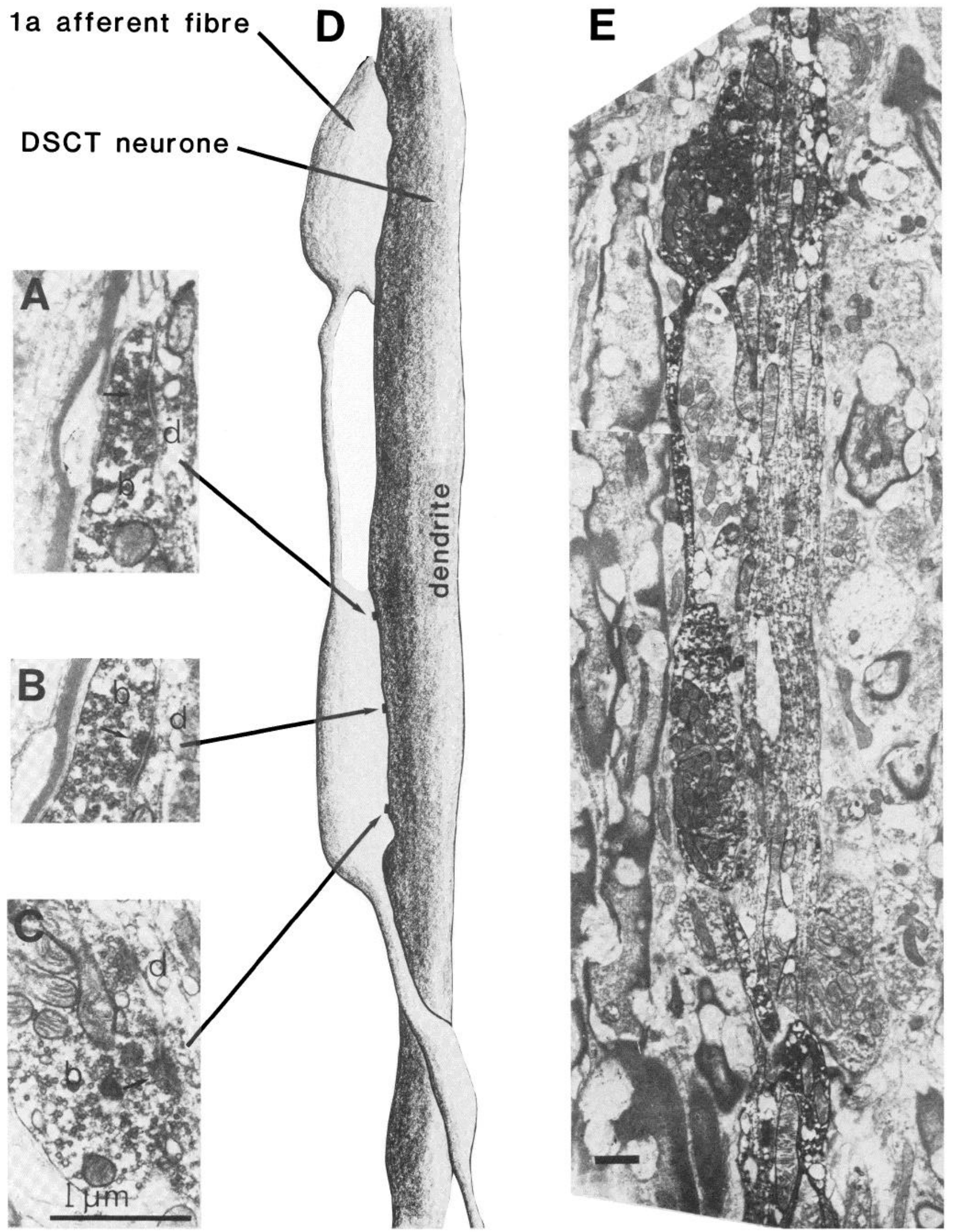

Figure 6. Details of the contacts between an HRP-labeled la bouton (same bouton as shown in Fig. 5) and the HRP-labeled dendrite of a DSCT neuron. Synaptic specializations (arrows in $A, B$, and $C ; d$, dendrite; $b$, bouton) were found in the regions indicated by the lines joining $A, B$, and $C$ to the drawing shown in $D$. This drawing was constructed from serial sections and is drawn to the same scale as the photomontage shown in $E$. Calibration bar in $E$ is 1.0 

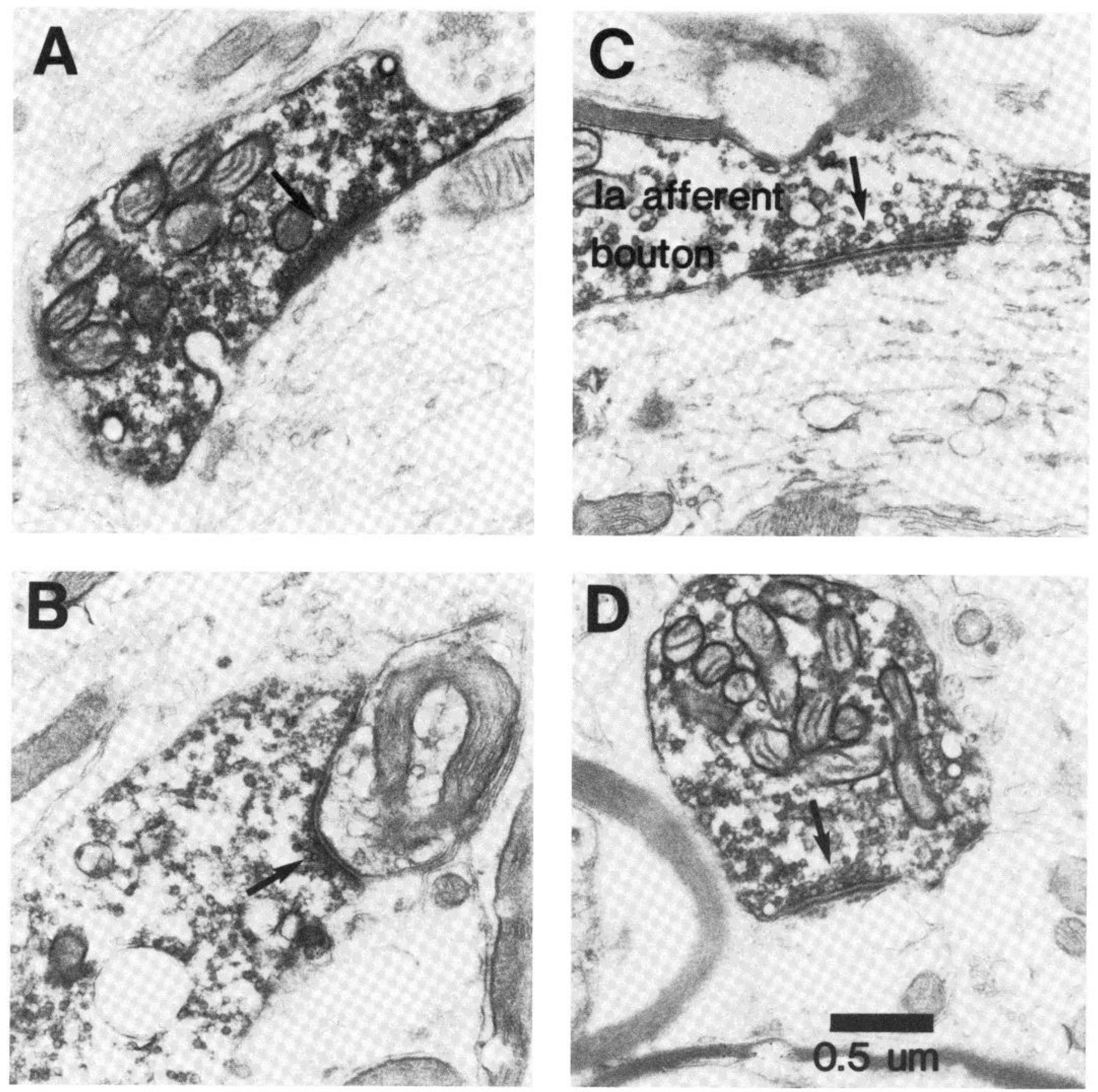

Figure 7. Contacts formed by HRP-labeled la boutons $(A$ to $D)$ within Clarke's column. Synaptic specializations are indicated by arrows. Note the array of postsynaptic dense bodies opposite the presynaptic thickenings in $C$ and $D$.

an HRP-labeled la afferent fiber and dendrites of the same labeled DSCT neuron.

The synaptic boutons were of both terminal (Fig. 2C) and en passant (Fig. 2, $A$ and $B$ ) types. The bouton in Figure 2, $A$ and $B$, is of the en passant type and can be seen arising from a node of Ranvier and making connection with an HRP-labeled dendrite of the DSCT neuron. The appearance of this synapse is similar to the "outpocketing" synapses observed by Uchizono (1975, e.g., Fig. 128 ) in the cat spinal cord. In Figure $2 B$, two regions of synaptic specialization can be seen, as evidenced by pre- and postsynaptic thickenings and presynaptic clusters of vesicles. Serial examination revealed that these were separate specializations rather than sections of the same specialization.
Figures 3 and 4 illustrate the connections made between another serially sectioned la bouton and the HRP labeled DSCT neuron. Figure 3 shows a sequence $(A$ to $J)$ of photomicrographs of the bouton and the contacted dendrites. It appears that two small diameter branches (indicated by a single and a double arrow, respectively) emerge from a larger dendrite. Such branchlets are frequently observed arising from both proximal and distal dendrites of intracellularly labeled (HRP) DSCT neurons (Houchin et al., 1983; see also Fig. 1; personal observation). The bouton itself is of the en passant rather than the terminal type as the (myelinated) axon is evident on both sides of the bouton, labeled in Figure $3, C$ and $H$. The la bouton formed single synaptic specializations with both of the HRP-labeled branchlets as shown in detail in Figure $4, B$ and $C$. 

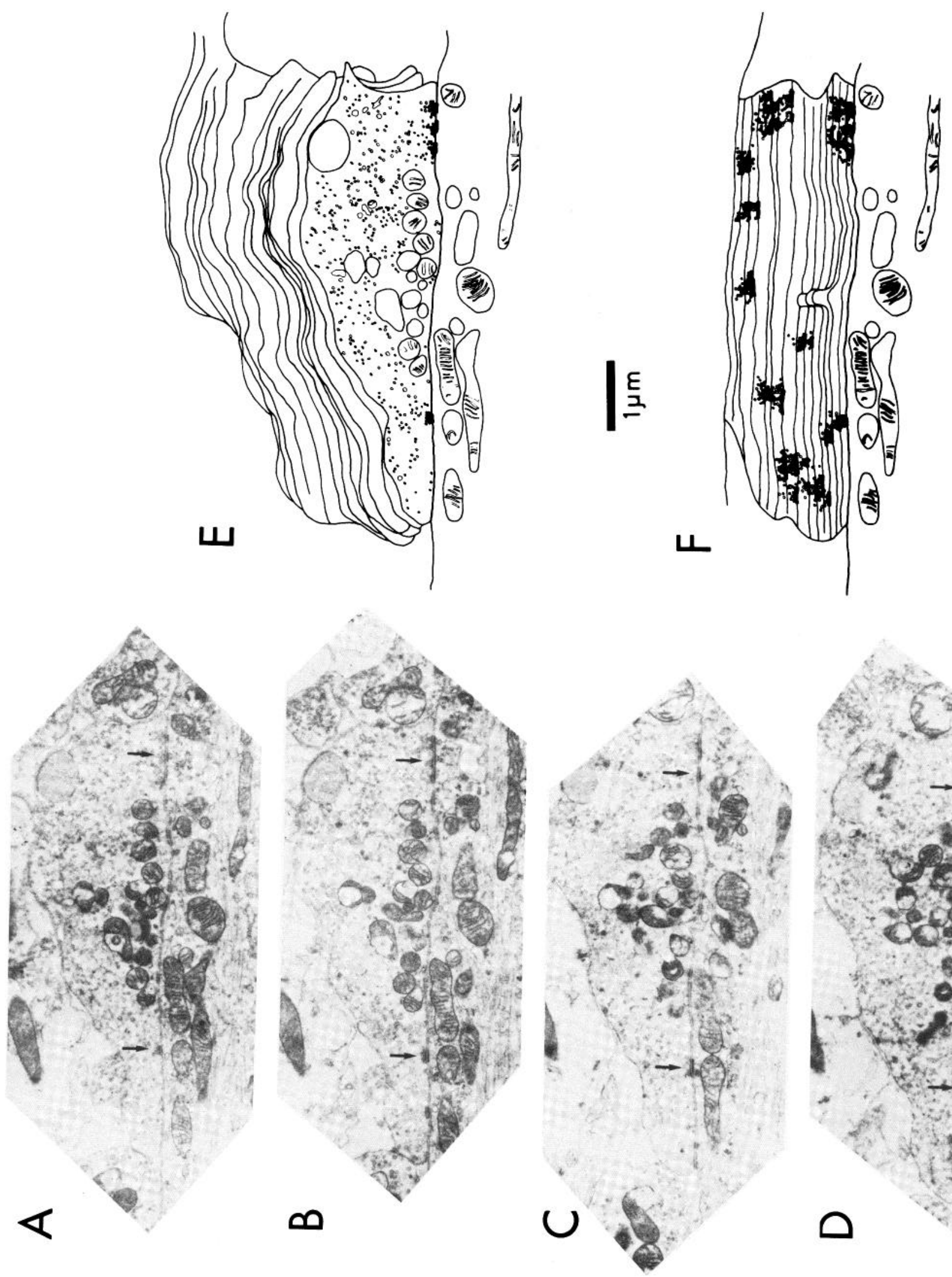

웅

a हो 긍 $\frac{5}{0}$ o 4 先

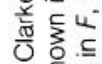
$\subseteq \frac{1}{\infty}$ 등드는 응듕 들 काष
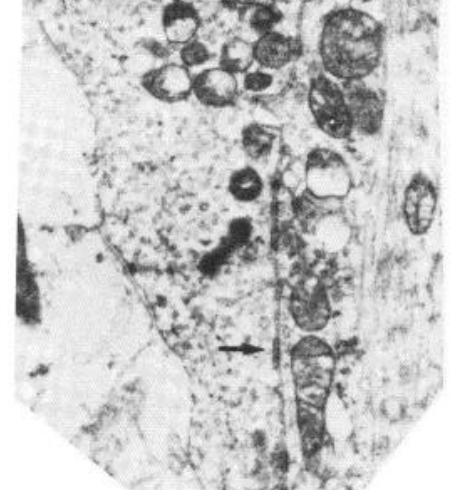
ธ。 혼 뜽 क ल

क्ष 틍휴 등 긍 을 응 हू 还 $\infty$ 음음

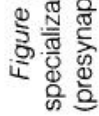


$\Delta$

B

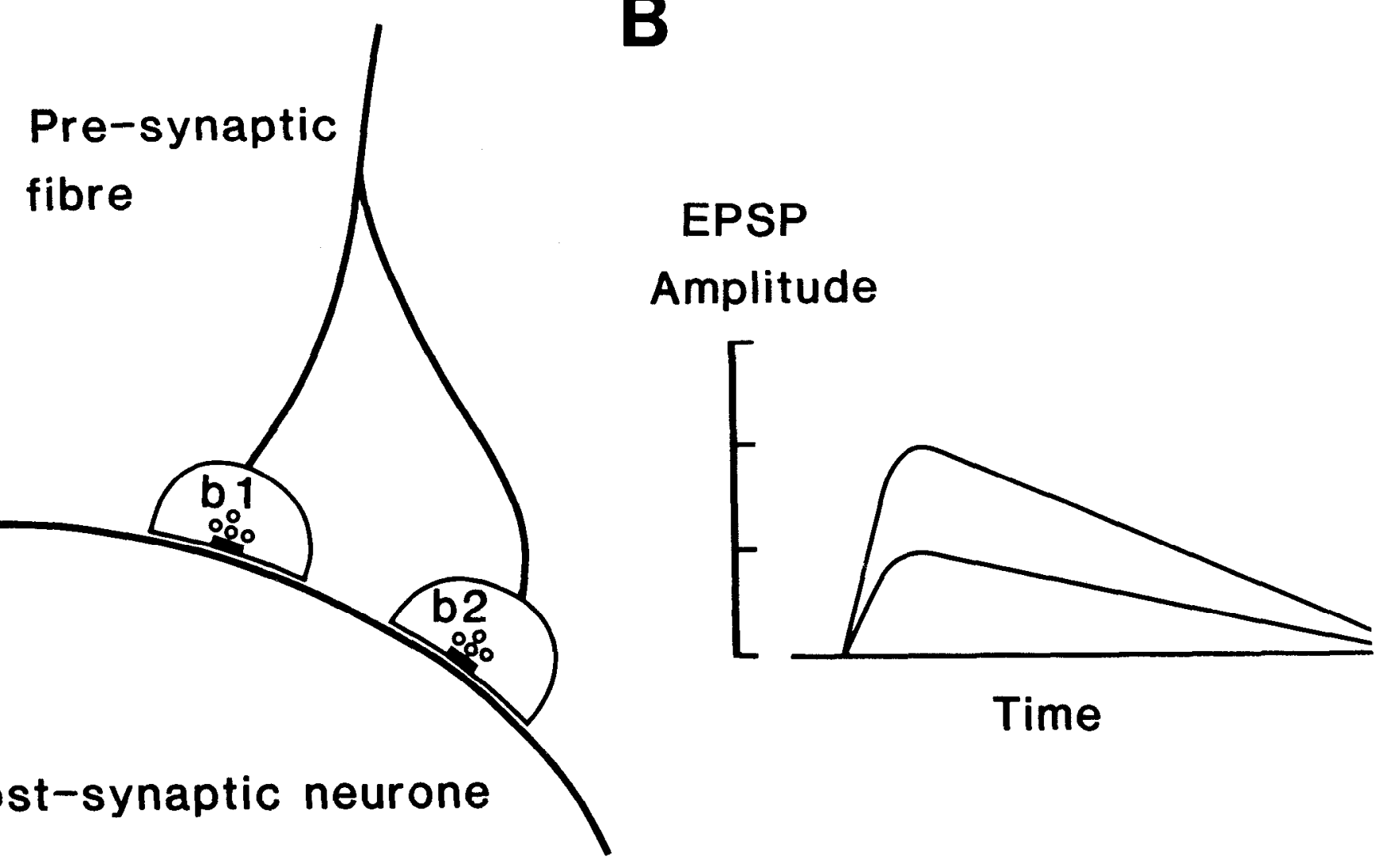

6

Pre-synaptic fibre

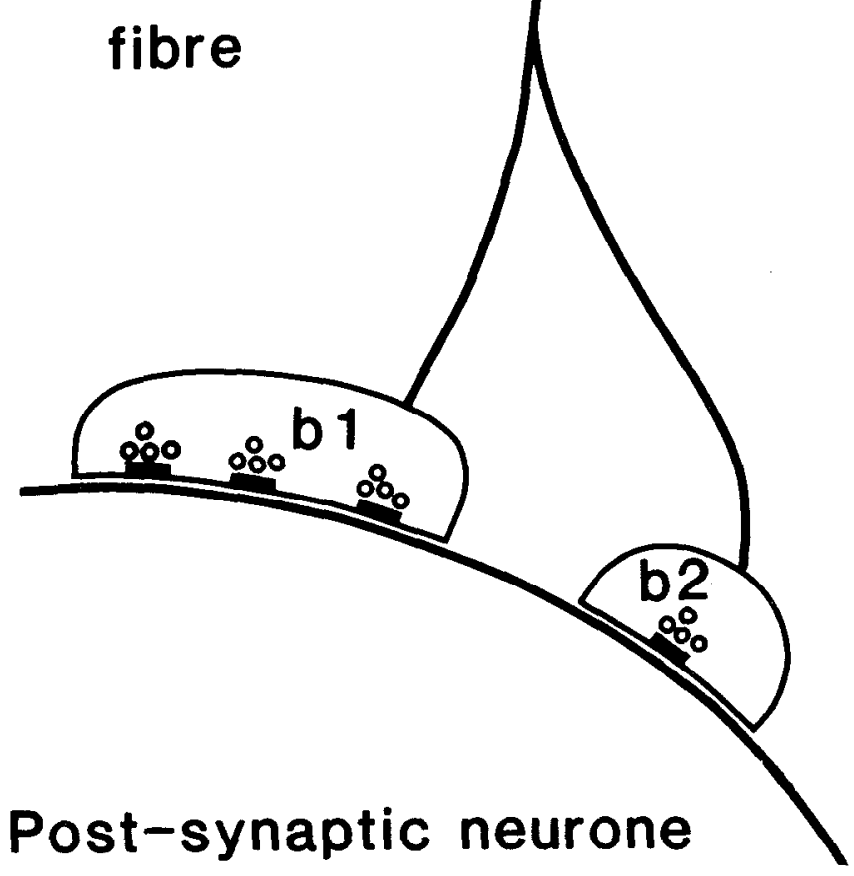

EPSP

Amplitude

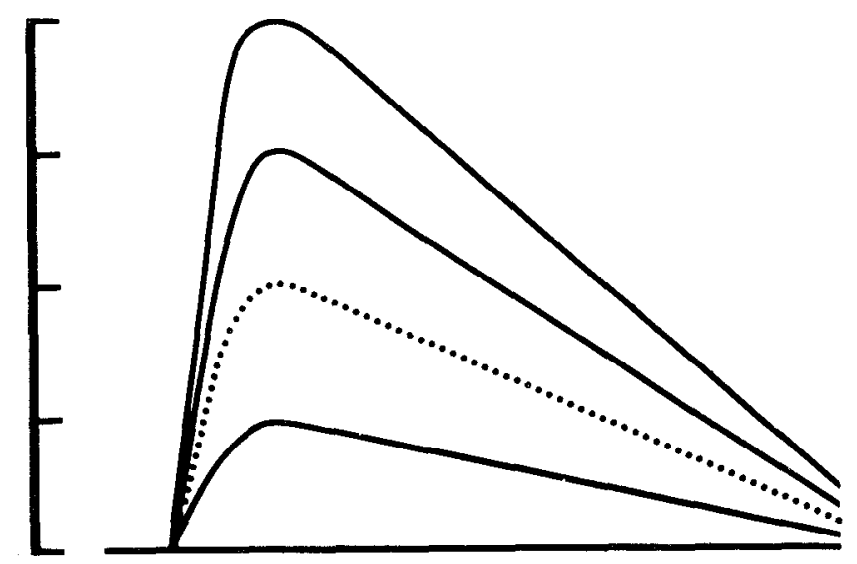

Time

Figure 9. Summary of two possible mechanisms of synaptic transmission in the spinal cord. See the text for explanation. 
In addition, the bouton contacted a small unlabeled structure, shown in Figure $4 A$ (asterisk). The approximate region of the bouton in which the contacts were found is indicated by the joining lines on the single section illustrated in Figure $4 D$ (only the postsynaptic dendrite shown in Fig. $4 B$ is visible in this single section).

Another HRP-labeled la bouton contacting the HRP-labeled DSCT neuron is illustrated in Figures 5 and 6 . Figure 5 shows a sequence of sections $(A$ to $H)$ through the la bouton. Three widely separated synaptic spccializations wore found in this bouton, and are indicated by arrows in Figure $5, B, D$, and $E$. These synaptic specializations are shown in detail in Figure $6, A$ to $C$, which also shows a threedimensional drawing which was reconstructed from serial sections of the bouton and the contacted dendrite. Figure $5 E$ shows a photomontage of the bouton and dendrite. The la afferent can be seen to enter and leave the bouton, which forms an en passant connection with the labeled dendrite.

Electron microscopy of la boutons in Clarke's column. Figure 7 illustrates synaptic specializations found in boutons arising from la fibers intracellularly labeled with HRP. The specializations varied in size for all la boutons examined from 0.3 to $1.0 \mu \mathrm{m}$ in diameter (longest axis). In two cases (Fig. 7, $C$ and $D$ ) a regular array of postsynaptic dense bodies was observed, similar to those associated with the M-bouton found on motoneurons (Conradi, 1969). All of the la boutons examined contained round, agranular vesicles, and the synaptic contacts formed were of the asymmetrical type (Colonnier, 1968). The size of la boutons examined varied over a considerable range, from 1.6 to $11.5 \mu \mathrm{m}$ long and 0.6 to $2.1 \mu \mathrm{m}$ wide. The number of synaptic specializations found in la boutons varied from one to four.

Electron microscopy of a "giant" bouton in Clarke's column. The light microscopic observations on la fibers in Clarke's column indicated that these fibers give rise to "giant" terminals up to $20 \mu \mathrm{m}$ long (Tracey and Walmsley, 1982, 1984). However, our limited sample of serially sectioned la boutons in this study did not allow us to examine such large boutons. To gain further information about these giant boutons, we have reconstructed an unlabeled giant bouton found within Clarke's column (Fig. 8), with the additional advantage that presynaptic elements were not obscured by the HRP-DAB reaction product found in the labeled boutons.

Reconstruction from $100-\mathrm{nm}$ serial sections revealed that this bouton formed nine synaptic contacts with a large dendrite. The long axis of this dendrite was parallel to the long axis of the bouton. The size of the postsynaptic densities varied from 0.2 to $0.7 \mu \mathrm{m}$ in diameter.

The three largest postsynaptic densities appeared as very irregular shapes similar to structures observed in rat cortex (Peters and Kaisermann-Abramof, 1969) and dog cortex (Cohen and Siekiewitz, 1978). A single section through such an irregularly shaped specialization (e.g., Fig. 8A) could be misinterpreted as multiple synaptic specializations. Serial reconstruction is obviously necessary to resolve such a problem (Fig. 8, $E$ and F).

\section{Discussion}

The results from this study confirm and extend previous light and electron microscopic studies on the synaptic connections between primary afferent fibers and DSCT neurons in Clarke's column (Rethelyi, 1970; Ilouchin et al., 1983; Tracey and Walmsley, 1982, 1984).

We have studied these connections using serial electron microscopy of afferents and DSCT neurons which were positively identified by direct intracellular injection of HRP. Single la afferent fibers give rise to a wide range of sizes of synaptic boutons in Clarke's column. Results from the present study show that an individual la bouton may contain multiple synaptic specializations, which was verified by detailed examination of serial sections. A single bouton could make contact with one or several postsynaptic profiles. An example was illustrated in which an HRP labeled la bouton formed synaptic contacts with two separate dendritic branches of the same HRP-labeled
DSCT neuron, in addition to a third contact with an unlabeled profile, presumably the dendrite of a neighboring neuron. In our study all la boutons contained agranular, spherical vesicles and formed contacts of an asymmetrical type. Several of the HRP-labeled la boutons exhibited a regular array of postsynaptic dense bodies, a characteristic feature of the M-boutons on motoneurons (Conradi, 1969). Such postsynaptic dense bodies have not been found, however, in electron microscopy of HRP-identified la fiber contacts with motoneurons (Conradi et al., 1983; Гyffe and Light, 1984).

A number of previous studies on synaptic transmission in the central nervous system have attempted to relate fluctuations in EPSP amplitude to transmitter release from synaptic boutons. Figure 9 illustrates schematically two proposals which have emerged from these studies. Figure $9 A$ illustrates the connection between a presynaptic fiber and a postsynaptic neuron. Two boutons, each containing a single transmitter release site, contact the neuron. Stimulation of the presynaptic fiber may result in no transmitter release from either release site, release from either bouton b1 or $b 2$, or release from both $b 1$ and $b 2$. The resulting EPSP may fluctuate between the three levels indicated in Figure $9 B$, depending on the probabilities of transmitter release from b1 and b2. In such a scheme each bouton contains only a single transmitter release site and it is not possible to decide whether there is a relationship between the fluctuations and the number of release sites or the number of boutons. Such a situation exists at an inhibitory synaptic connection on the Mauthner cell in the goldfish (Korn et al., 1981). Studies on the fluctuations in single group la fiber EPSPs evoked in cat spinal motoneurons have led to the hypothesis that each increment in an EPSP is the symaplic polential generaled by a single bouton (Redman and Walmsley, 1981, 1983b).

Studies of EPSPs evoked in DSCT neurons by impulses in single primary muscle afferents have revealed that these EPSPS fluctuated in amplitude. In general, these single fiber EPSPs fluctuate between discrete amplitudes separated by a quantal increment (Tracey and Walmsley, 1984). The present study has shown that a single la bouton contacting a DSCT neuron may contain multiple transmitter release sites. Such a situation is represented in Figure $9, C$ and $D$. Two boutons contact the postsynaptic neuron with one bouton containing three transmitter release sites and the other bouton contains only a single release site. The EPSP resulting from transmission at a bouton containing multiple release sites would be larger than that from a bouton containing only a single release site. If the boutons b1 and b2 in Figure $9 \mathrm{C}$ acted as all-or-nothing release elements, then the fluctuations would be nonquantal, as illustrated in Figure 9D, excluding the dotted EPSP. However, no large increments in EPSP amplitude fluctuations have been found (Tracey and Walmsley, 1984), and this possibility is considered unlikely. A more probable mechanism is that transmitter release sites within a single bouton act independently. In the illustration shown in Figure $9, \mathrm{C}$ and $D$, the EPSP would fluctuate between five possible amplitude levels (including zero).

There are a number of factors which complicate the scheme presented in Figure 9, $C$ and $D$. The probability that transmitter will be released following arrival of an action potential in a synaptic bouton may be different from bouton to bouton. For example, some boutons may be subjected to presynaptic inhibition whereas others are not, and the amount of calcium entering one bouton may be different from another. In a bouton containing multiple transmitter release sites, the probability of release may not be the same for all release sites. Although quantal in nature, the fluctuation pattern of single la fiber EPSPs in motoneurons does not obey any standard statistical distribution, such as Poisson or binomial (Jack et al., 1981). Such distributions (Poisson or binomial) would be expected if the probability of transmitter release were identical for all release sites. It seems likely, therefore, that the EPSP fluctuation pattern observed for single la fiber EPSPs in DSCT neurons (and probably motoneurons) is due to a combination of all-or-nothing transmitter 
release from individual release sites, and that the probability of release may vary from release site to release site.

\section{References}

Adams, J. C. (1977) Technical considerations on the use of horseradish peroxidase as a neuronal marker. Neuroscience 2: 141-145.

Adams, J. C. (1981) Heavy metal intensification of DAB based HRP reaction product. J. Histochem. Cytochem. 29: 775.

Cohen, R. S., and P. Siekiewitz (1978) Form of the postsynaptic density. A serial section study. J. Cell. Biol. 78: 36-46.

Colonnier, M. (1968) Synaptic patterns on different cell types in the different laminae of the cat visual cortex. An electron microscope study. Brain Res. 9: $268-287$.

Conradi, S. (1969) Ultrastructure of dorsal root boutons on lumbosacral motoneurons of the adult cat, as revealed by dorsal root section. Acta Physiol. Scand. Suppl. 332: 85-115.

Conradi, S., S. Cultheim, L.Gollvik, and J. -O. Kellerth (1983) Electron microscopic observations on the synaptic contacts of group la muscle spindle afferents in the cat lumbosacral cord. Brain Res. 265: 31-39.

Fyffe, R. E. W. and A. R. I ight (1984) The ultrastructure of group la afferent fiber synapses in the lumbosacral spinal cord of the cat. Brain Res. 300: 201-209.

Houchin, J., D. J. Maxwell, R. E. W. Fyffe, and A. G. Brown (1983) Light and electron microscopy of dorsal spinocerebellar tract neurones in the cat: An intracellular horseradish peroxidase study. Q. J. Exp. Physiol. 68: 719732.

Jack, J. J. B., S. J. Redman, and K. Wong (1981) The components of synaptic potentials evoked in cat spinal motoneurones by impulses in single group la fibres. J. Physiol. (Lond.) 321: 65-96.

Korn, H., A. Triller, A. Mallart, and D. S. Faber (1981) Fluctuating responses at a central synapse: $n$ of binomial fit predicts number of stained presynaptic boutons. Science (N.Y.) 213: 898-901.

Mann, M. D. (1973) Clarke's column and the dorsal spinocerebellar tract. Brain Behav. Evol. 7: 34-83.
Peters, A., and I. R. Kaisermann-Abramof (1969) The small pyramidal neuron of the rat cerebral cortex. The synapses upon dendritic spines. Z. Zellforsch. Nilenos Aust. 100: 487-506.

Randic, M. M., V. Miletic, and A. D. Loewy (1981) A morphological study of cat dorsal spinocerebellar tract neurones after intracellular injection of horseradish peroxidase. J. Comp. Neurol. 198: 453-466.

Redman, S. J., and B. Walmsley (1981) The synaptic basis of the monosynaptic stretch reflex. Trends Neurosci. 4: 248-250.

Redman, S. J., and B. Walmsley (1983a) The time course of synaptic potentials evoked in cal spinal motoneurones at identified group la synapses. J. Physiol. (Lond.) 343: 117-133.

Redman, S. J., and B. Walmsley (1983b) Amplitude fluctuations in synaptic potentials evoked in cat spinal motoneurones at identified group la synapses. J. Physiol. (Lond.) 343: 135-145

Rethelyi, M. (1970) Ultrastructural synaptology of Clarke's column. Exp. Brain Res. 11: 159-174.

Saito, K. (1974) The synaptology and cytology of the Clarke cell in nucleus dorsalis of the cat: An electron microscopic study. J. Neurocytol. 3: 179197

Saito, K. (1979) Morphometrical synaptology of Clarke cells and of distal dendrites in the nucleus dorsalis: Electron microscopic study in the cat. Brain Res. 178: 233-249.

Szentagothai, J., and A. Albert (1955) The synaptology of Clarke's column. Acta Morphol. Hung. 5: 43-51.

Tracey, D. J., and B. Walmsley (1982) Anatomy of the connection between identified primary afferents and neurones of the dorsal spinocerebellar tract. Proc. Aust. Physiol. Pharmacol. Soc. 13: (2) 134P.

Tracey, D. J., and B. Walmsley (1984) Synaptic input from identified muscle afferents to neurones of the dorsal spinocerebellar tract in the cat. $J$. Physiol. (Lond.) 350: 599-614.

Uchizono, K. (1975) Excitation and Inhibition: Synaptic Morphology, Elsevier Scientific Publishing Co., Amsterdam.

Wienawa-Narkiewicz, E., B. Walmsley, and M. J. Nicol (1984) Electron microscopy of the synaptic connection between primary afferents and DSCT neurones. Neurosci. Lett. Suppl. 15: S63. 